Journal Afrika Statistika

Vol. 5, $\mathrm{N}^{\circ}$ 7, 2010, page 245-251.

\title{
A simple note on some empirical stochastic process as a tool in uniform L-statistics weak laws
}

\section{Gane Samb Lô}

LERSTAD, Université Gaston Berger, Saint-Louis, SENEGAL LSTA, Université Pierre et Marie Curie, Paris, FRANCE

Received 15 July 2010; Accepted 11 October 2010

Copyright (C) 2010, Journal Afrika Statistika. All rights reserved

Abstract. In this paper, we are concerned with the stochastic process

$$
\beta_{n}\left(q_{t}, t\right)=\beta_{n}(t)=\frac{1}{\sqrt{n}} \sum_{j=1}^{n}\left\{G_{t, n}(Y(t))-G_{t}\left(Y_{j}(t)\right)\right\} q_{t}\left(Y_{j}(t)\right),
$$

where for $n \geq 1$ and $T>0$, the sequences $\left\{Y_{1}(t), Y_{2}(t), \ldots, Y_{n}(t), t \in[0, T]\right\}$ are independent observations of some real stochastic process $Y(t), t \in[0, T]$, for each $t \in[0, T], G_{t}$ is the distribution function of $Y(t)$ and $G_{t, n}$ is the empirical distribution function based on $Y_{1}(t), Y_{2}(t), \ldots, Y_{n}(t)$, and finally $q_{t}$ is a bounded real function defined on $\mathbb{R}$. This process appears when investigating some time-dependent L-Statistics which are expressed as a function of some functional empirical process and the process (A). Since the functional empirical process is widely investigated in the literature, the process reveals itself as an important key for L-Statistics laws. In this paper, we state an extended study of this process, give complete calculations of the first moments, the covariance function and find conditions for asymptotic tightness.

Résumé. Nous nous interéssons dans ce papier au processus stochastique suivant

$$
\beta_{n}\left(q_{t}, t\right)=\beta_{n}(t)=\frac{1}{\sqrt{n}} \sum_{j=1}^{n}\left\{G_{t, n}(Y(t))-G_{t}\left(Y_{j}(t)\right)\right\} q_{t}\left(Y_{j}(t)\right),
$$

où $n \geq 1, T>0,\left\{Y_{1}(t), Y_{2}(t), \ldots, Y_{n}(t), t \in[0, T]\right\}$ sont des observations indépendantes d'un certain processus réel $\{Y(t), t \in[0, T]\}$, pour tou $t \in[0, T], G_{t}$ est la fonction de répartition de $Y(t), G_{t, n}$ est la fonction de répartition empirique basée sur l'échantillon $Y_{1}(t), Y_{2}(t), \ldots, Y_{n}(t)$, et enfin $q_{t}$ est une fonction réelle bornée définie sur $\mathbb{R}$. Ce processus apparaît lors de l'investigation de L-Statistiques dépendant du temps, que l'on peut exprimer comme une somme d'un processus empirique fonctionnel et d'un processus de type (A). Puisque les processus empiriques fonctionnels sont largement étudiés, l'étude du processus (A) devient incontournable pour décrire le comportement asymptotique de L-Statistiques. Dans ce papier, nous menons une étude complète du processus (A), calculons ses premiers moments, sa fonction de covariance limite ainsi que les conditions de tension asymptotique. De même la somme d'un processus empirique et d'un processus de type (A) et la somme de deux processus de même type (A), lorsqu'ils tous basés sur le même échantillon temporel, sont largement décrites.

Key words: Empirical processes; Order Statistics; L-statistics.

AMS 2000 Mathematics Subject Classification : 62E20, 62F12, 62G20, G2G05.

\section{Introduction}

In this paper, we are concerned with the uniform weak laws of a special process occurring in some research areas like Actuarial Sciences when measuring heavy losses, Welfare Sciences when measuring inequality coefficients and 
G.S. Lô, Journal Afrika Statistika, Vol. 5, N 7,2010 , page 245-251.

A simple note on some empirical stochastic process as a tool in uniform L-statistics weak laws

poverty indices. As well, it may be applied for general L-statistics. In order to define it, let $n \geq 1$ be a positive integer and $Y_{1}, Y_{2}, \ldots, Y_{n}$ independent and identically distributed random variables with values in $\ell^{\infty}([0, T])$, the space of real bounded functions defined on time space $[0, T]$, where $T$ is a fixed positive real number. This means that the observations depend on the time $t \in[0, T]$, so that we may also write them in the form

$$
\left\{Y_{1}(t), Y_{2}(t), \ldots, Y_{n}(t), t \in[0, T]\right\}
$$

and we represent the order statistics, when needed, by $Y_{1, n}(t) \leq Y_{2, n}(t) \leq \ldots \leq Y_{n, n}(t)$. Now let $k \geq 1$ and $0<$ $t_{1}<t_{2}<\ldots<t_{k} \leq T, G_{t_{1}, t_{2}, \ldots, t_{k}}$ will stand for the distribution function of $\left(Y_{j}\left(t_{1}\right), Y_{j}\left(t_{2}\right), \ldots, Y_{j}\left(t_{k}\right)\right)^{t}$. Also, for each $t \in[0, T]$, we denote by $G_{t, n}$ the empirical distribution function based on the sample $Y_{1}(t), Y_{2}(t), \ldots, Y_{n}(t)$, that is, for each $x \in R$,

$$
n G_{t, n}(x)=\sum_{j=1}^{n} 1_{\left(Y_{j}(t) \leq x\right)}
$$

From now, we suppose that all the random variables used here are defined on the same probability space $(\Omega, A, P)$. We are now able to introduce the process

$$
\beta_{n}\left(q_{t}, t\right)=\beta_{n}(t)=\frac{1}{\sqrt{n}} \sum_{j=1}^{n}\left\{G _ { t , n } \left(Y(t)-G_{t}\left(Y_{j}(t)\right\} q_{t}\left(Y_{j}(t)\right),\right.\right.
$$

where for each $t \in[0,1], q_{t}: R \longmapsto R$ is a measurable bounded function. For $q \equiv 1$, we write it $B_{n}^{*}(t)=\beta_{n}(1, t)$ and called it as the simple process. This process $\left\{\beta_{n}(t), t \in[0, T]\right\}$ may appear when dealing with time-dependant L-Statistics of the form

$$
J_{n}(t)=\frac{1}{n} \sum_{j=1}^{Q_{n}(t)} c(j / n) q_{0}\left(Y_{j, n}(t)\right),
$$

where $c(\cdot)$ (resp. $\left.q_{0}(\cdot)\right)$ is a function defined on $[0,1]$ (resp. $R$ ) and where for each fixed $t \in[0, T], Z(t)>0$ is some threshold such that $Y_{Q_{n}, n}(t) \leq Z(t)<Y_{Q_{n}+1, n}(t)$. By denoting $R_{j, n}(t)$ the rank statistics of $Y_{j}(t)$, (2) may be written, when the distribution functions $G_{t}$ are continuous, as

$$
\begin{gathered}
J_{n}(t)=\frac{1}{n} \sum_{j=1}^{n} c\left(R_{j, n}(t) / n\right) q\left(Y_{j}(t)\right) \mathbb{I}\left(Y_{j}(t) \leq Z(t)\right) \\
=\frac{1}{n} \sum_{j=1}^{n} c\left(G_{t, n}\left(Y_{j}(t)\right) q_{1}\left(Y_{j}(t)\right)\right.
\end{gathered}
$$

where $q_{1}(Y(t))=q_{0}(Y(t)) I(Y(t) \leq Z(t))$. Under some conditions (see Lo [5]), (2) may be uniformly approximated by the representation, as $n \rightarrow \infty$,

$$
\begin{aligned}
J_{n}(t) & =\frac{1}{n} \sum_{j=1}^{n} c\left(G_{t}\left(Y_{j}(t)\right) q_{1}\left(Y_{j}(t)\right)\right. \\
+ & \frac{1}{n} \sum_{j=1}^{n}\left\{G _ { t , n } \left(Y(t)-G_{t}\left(Y_{j}(t)\right\} c^{\prime}\left(G_{t}\left(Y_{j}(t)\right) q_{1}\left(Y_{j}(t)\right)+o_{P}^{*}\left(n^{-1 / 2}\right),\right.\right.\right.
\end{aligned}
$$

where $c^{\prime}$ is the derivative function of $c$, and $u_{n}^{*}=o_{P}^{*}(1)$ stands for the convergence to zero in outer-probability, that is there exists a sequence of random variables $u_{n}$ converging to zero in probability as $n \rightarrow+\infty$ and $\left\|u_{n}^{*}\right\| \leq\left\|u_{n}\right\|$ for $n \geq 1$. Putting

$$
J(t)=\mathbb{E} c\left(G_{t}\left(Y_{j}(t)\right) q_{1}\left(Y_{j}(t)\right)=\int_{\mathbb{R}} c\left(G_{t}(y)\right) q_{1}(y) d G_{t}(y)\right.
$$

we have, for $q_{t}(\cdot)=c^{\prime}\left(G_{t}(\cdot) q_{1}(\cdot)\right.$, as $n \rightarrow \infty$,

$$
\sqrt{n}\left(J_{n}(t)-J(t)\right)=\alpha_{n}(t)+\beta_{n}\left(q_{t}, t\right)+o_{P}(1),
$$

where

$$
\alpha_{n}(t)=\frac{1}{\sqrt{n}} \sum_{j=1}^{n}\left\{c \left(G_{t}\left(Y_{j}(t)\right) q_{1}\left(Y_{j}(t)\right)-\mathbb{E} c\left(G_{t}\left(Y_{j}(t)\right) q_{1}\left(Y_{j}(t)\right)\right\}\right.\right.
$$


and this is nothing else but the functional empirical process $G_{n}$ so that

$$
\alpha_{n}(t)=\mathbb{G}_{n}\left(g_{t}\right)=\frac{1}{\sqrt{n}} \sum_{j=1}^{n}\left\{g_{t}\left(Y_{j}\right)-\mathbb{E} g_{t}\left(Y_{j}\right)\right\},
$$

where $g_{t}$ is the real function defined on $\ell^{\infty}([0, T])$ satisfying

$$
g_{t}(x)=c\left(G_{t}(x(t)) q_{1}(x(t)), x \in \ell^{\infty}([0, T]) .\right.
$$

Statistics like (2) thus are present in many situations in connection with L-Statistics (see Helmers and Ruymgaart [1], Helmers et al. [2,3]) and naturally occur is Actuarial Sciences and in inequality measures (see Puri et al. [4]), and more recently in poverty measures (see Lo [5], Lo et al. [8]). In all these fields, we may be faced not to find simple asymptotic normality results, but to derive uniform asymptotic laws for the time-dependant statistics (with the parameter $t \in[0, T])$ and functional asymptotic laws with respect to the class of functions $F=\left\{\left(g_{t}, q_{t}\right), t \in[0, T]\right\}$.

This motivated us to undertake a special study of $\beta_{n}$ and its connection with the empirical process as general key tools. This study needs much calculations that may be superfluous in each particular application. We thus aim to characterize this process here and present our results as general tools to be used further in statistical works as packages. In all the paper, we suppose that the distribution functions $G_{t}$ are continuous and increasing.

Since the calculations related to this study are tremendous, we are going to give here the characteristics of the process. Examples of computations that lead to the results stated here are given in the beginning of the proof of the first theorem while the full paper are given in Lo and Sall [6].

The paper is organized as follows. We entirely describe the weak law the process in Section 2. In Section 3, the weak law of the sum of a process of type (1) with a functional empirical process is given while Section 3 is devoted to the weak law of a couple of statistics of type (1). The paper is finished by a conclusion.

\section{Law of the general process}

We now consider the process

$$
\beta_{n}^{*}(t)=\sqrt{n} \beta_{n}(t)=\sum_{j=1}^{n}\left\{G_{t, n}\left(Y_{j}(t)\right)-G_{t}\left(Y_{j}(t)\right)\right\} q_{t}\left(Y_{j}(t)\right) .
$$

Before we present our main result, define

$$
\begin{gathered}
g(q, t, s)=\int\left(\int_{x \geq u} q_{t}(x) G_{t}(x)\right)\left(\int_{y \geq v} q_{s}(y) G_{t}(y)\right) d G_{t, s}(u, v) \\
c_{2}(t)=\int\left(\int_{x \geq u} q_{t}(x) G_{t}(x)\right)^{2} d G_{t}(u)
\end{gathered}
$$

and this convention, for a function $h$,

$$
\mathbb{E}_{t} h=\int h(u) d G_{t}(u)
$$

Theorem 1. If there is a universal constant $K_{0}$, such that there exists $\delta>0$,

$$
\begin{gathered}
|s-t| \leq \delta \Longrightarrow \mid 2\left(c_{2}(t)-g(q, t, s)\right) \\
+\left\{\left(\mathbb{E}_{t} G_{t} q_{t}\right)\left(\mathbb{E}_{s} G_{s} q_{t}\right)-\left(\mathbb{E}_{t} G_{t} q_{t}\right)^{2}\right\}\left|\leq \frac{3}{2} K_{0}\right| s-\left.t\right|^{1+r},
\end{gathered}
$$

then $\left\{\beta_{n}(t), 0 \leq t \leq T\right\}$ converges to a $\ell^{\infty}([0, T])$-Gaussian process with covariance function

$$
\Gamma_{1}\left(q_{t}, q_{s}, s, t\right)=g(q, t, s)-\left(\mathbb{E}_{t} G_{t} q_{t}\right)\left(\mathbb{E}_{s} G_{s} q_{s}\right) .
$$

Remark 1. As announced, we will give in the beginning of the proof of this theorem examples of computations needed in proving the results of these paper. Full, detailed and complete ones are stated in Lo and Sall [6]. 
G.S. Lô, Journal Afrika Statistika, Vol. 5, N 7,2010 , page 245-251.

Proof. Let

$$
\beta_{n}^{*}(t)=\sqrt{n} \beta_{n}(t) .
$$

We begin to calculate the two first moments and the covariance function.

Mean calculation. One has

$$
\mathbb{E} \beta_{n}^{*}(t)=\mathbb{E} \sum_{j=1}^{n} G_{t, n}\left(Y_{j}(t)\right) q_{t}\left(Y_{j}(t)\right)-n\left(\mathbb { E } q _ { t } ( Y ( t ) ) \left(G_{t}(Y(t)) .\right.\right.
$$

But

$$
n G_{t, n}\left(Y_{j}(t)\right) q_{t}\left(Y_{j}(t)\right)=q_{t}\left(Y_{j}(t)\right)+\sum_{h \neq j} 1_{\left(Y_{h}(t) \leq Y_{j}(t)\right)} q_{t}\left(Y_{j}(t)\right)
$$

and

$$
\begin{gathered}
\mathbb{E} n G_{t, n}\left(Y_{j}(t)\right) q_{t}\left(Y_{j}(t)\right)=\mathbb{E} q_{t}(Y(t))+(n-1) \int q_{t}(u) d G_{t}(u) \int_{x \geq u} d G_{t}(x) \\
=\mathbb{E}_{t} q_{t}+(n-1) \int G_{t}(u) q_{t}(u) d G_{t}(u) .
\end{gathered}
$$

Recall the convention $E_{t} b=E(b(Y(t)))$. We get

$$
\mathbb{E} G_{t, n}\left(Y_{j}(t)\right) q_{t}\left(Y_{j}(t)\right)=\frac{\mathbb{E}_{t} q_{t}-\mathbb{E}_{t} q_{t} G_{t}}{n}+\mathbb{E}_{t} q_{t} G_{t} .
$$

This gives

$$
\mathbb{E} \beta_{n}^{*}(t)=\mathbb{E}_{t} q_{t}-\mathbb{E}_{t} q_{t} G_{t}
$$

and

$$
\mathbb{E} \beta_{n}(t)=\left(\mathbb{E}_{t} q_{t}-\mathbb{E}_{t} q_{t} G_{t}\right) / \sqrt{n} \rightarrow 0 .
$$

Variance calculation. Direct calculations like the previous give :

$$
\mathbb{E} \beta_{n}(t)^{2}=c_{2}(t)-\left(\mathbb{E}_{t} G_{t} q_{t}\right)^{2}+\frac{K_{1}(t, s)}{n},
$$

where $K_{1}(t, s)$ is uniformly bounded. Before we arrive at the covariance function. We should observe that for $q_{t}=1$, then $c_{2}=1 / 3,\left(E_{t} G_{t} q\right)^{2}=1 / 4$ and

$$
c_{2}(t)-\left(\mathbb{E}_{t} G_{t} q_{t}\right)^{2}=1 / 12
$$

Covariance calculations. We also have

$$
\begin{aligned}
\mathbb{E} \beta_{n}(t) \beta_{n}(s) & =g(q, t, s)-\left(\mathbb{E}_{t} G_{t} q_{t}\right)\left(\mathbb{E}_{s} G_{s} q_{s}\right)+\frac{K_{2}(n, t, s)}{n} . \\
& =\Gamma_{1}\left(q_{t}, q_{s}, t, s\right)+\frac{K_{2}(n, t, s)}{n},
\end{aligned}
$$

where $K_{2}(n, t, s)$ is uniformly bounded in $(n, t, s)$. We finish to remark that for $s=t$, we get

$$
\mathbb{E} \beta_{n}(t)^{2} \sim c_{2}(t)-\left(E_{t} G_{t} q\right)^{2} .
$$

We now consider the increments of $\beta_{n}(t)$.

Increments calculations.

Recall that

$$
E \beta_{n}(t)^{2}=c_{2}(t)-\left(E_{t} G_{t} q\right)^{2}+\frac{K_{1}(n, t)}{n} .
$$

This gives

$$
E\left(\beta_{n}(t)-\beta_{n}(s)\right)^{2}=2\left(c_{2}(t)-g(q, t, s)\right)+\left\{\left(E_{t} G_{t} q\right)\left(E_{s} G_{s} q\right)-\left(E_{t} G_{t} q\right)^{2}\right\}+\frac{K_{3}(n, t, s)}{n} .
$$

Proofs of the weak convergence. 
We always begin to show the weak convergence of the finite-distribution of $\beta_{n}(\cdot)$ that is

$$
\beta_{n}\left(t_{1}, \ldots, t_{k}, a\right)=\sum_{j=1}^{k} \alpha_{j} \beta_{n}\left(t_{j}\right)=\frac{1}{\sqrt{n}} \sum_{s=1}^{k} a_{s} \sum_{j=1}^{n}\left\{G_{t_{s}, n}\left(Y_{j}\left(t_{s}\right)\right)-G\left(Y_{t_{s}}\right)\right\} q_{t_{s}}\left(Y_{j}\left(t_{s}\right)\right) .
$$

$0<t_{0}<t_{1}<\ldots<t_{k} \leq T, a=\left(a_{1}, \ldots, a_{k}\right)^{t} \in R^{k}$. We have

$$
\beta_{n}(t)=\int_{0}^{1} \sqrt{n}\left(s-V_{t, n}(s)\right) q_{t}\left(G_{t}^{-1}(s)\right) d s+O_{P}(1 / \sqrt{n})=N_{n}^{*}\left(q_{t}, t\right)+O_{P}(1 / \sqrt{n}) .
$$

The finite distribution is established by using Lemma 1 below and its application in section 5 . The covariance function of the limiting process is

$$
\Gamma_{1}\left(q_{t_{i}}, q_{t_{j}}, t_{i}, t_{j}\right)=\lim _{n \rightarrow \infty} \mathbb{C o v}\left(N_{n}^{*}\left(q_{t_{i}}, t_{i}\right), N_{n}^{*}\left(q_{t_{j}}, t_{j}\right)\right)
$$

which, by (6), is

$$
\Gamma_{1}\left(q_{t_{i}}, q_{t_{j}}, t_{i}, t_{j}\right)=\lim _{n \rightarrow \infty} \operatorname{Cov}\left(\beta_{n}(t), \beta_{n}(s)\right)
$$

Finally (4), (5) together prove the asymptotic tightness of $\beta_{n}$ via Lemma 1 in Sall and Lo [11] and Example 2.2.12 in van der Vaart and Wellner [10].

\section{Addition of the processes and an empirical process}

In many situations, the asymptotic law of the studied statistics is achieved in a sum of our process and an empirical process of the form

$$
\gamma_{n}=\alpha_{n}+\beta_{n}
$$

where

$$
\gamma_{n}(t)=\frac{1}{\sqrt{n}} \sum_{j}\left(g_{t}(Y(t))-\eta(t)\right)+\frac{1}{\sqrt{n}} \sum_{j}\left\{G_{t, n}\left(Y_{j}(t)\right)-G_{t}\left(Y_{j}(t)\right)\right\} q_{t}\left(Y_{j}(t)\right) .
$$

In such cases, what is the covariance structure of the limiting process? We have this

Proposition 1. If each of the processes $\gamma_{n}, \alpha_{n}$ and $\beta_{n}$ converges in finite-distributions and is asymptotically tight, then covariance function of the limiting Gaussian process of $\gamma_{n}$ is

$$
\Gamma(t, s)=\Gamma_{1}\left(q_{t}, q_{s}, t, s\right)+\Gamma_{2}(t, s)+\gamma(t, s),
$$

with

and

$$
\begin{gathered}
\Gamma_{2}(t, s)=\int\left(\bar{g}_{t}(x)-\eta(t)\right)\left(\bar{g}_{s}(y)-\eta(s)\right) d G_{t, s}(x, y), \\
\Gamma_{2}\left(q_{t}, q_{s}, t, s\right)=g(q, t, s)-\left(\mathbb{E}_{t} G_{t} q_{t}\right)\left(\mathbb{E}_{s} G_{s} q_{s}\right), \\
g\left(q_{t}, q_{s}, t, s\right)=\int\left(\int_{x \geq u} q_{t}(u) d G_{t}(u)\right)\left(\int_{x \geq v} q_{t}(v) d G_{t}(v)\right) d G_{t, s}(u, v)
\end{gathered}
$$

with

$$
\gamma(t, s)=\gamma_{1}(t, s)+\gamma_{1}(s, t)
$$

$$
\gamma_{1}(t, s)=\int \bar{g}_{t}(u)\left(\int_{x \geq u} q(x) d G_{s}(u)\right) d G_{t, s}(u, v)
$$

Remark 2. We are not interesting here by complete results. We only intend to show how the process intervenes in general L-Statistics and to give the covariance function. In each particular,we will have to prove the finite-distribution convergence and the tightness of the components of such processes.

Proof. If the hypotheses of the proposition hold, the limiting covariance function is performed through the formula

$$
\begin{aligned}
\gamma_{n}(t) \gamma_{n}(s) & =\left(\alpha_{n}(t)+\beta_{n}(t)\right)\left(\alpha_{n}(s)+\beta_{n}(s)\right) \\
& \left.=\alpha_{n}(t) \alpha_{n}(s)+\alpha_{n}(t) \beta_{n}(s)+\beta_{n}(t)\left(\alpha_{n}(s)+\beta_{n}(t) \beta_{n}(s)\right)\right) .
\end{aligned}
$$

By computing the expectation of each of them, we arrive at

$$
\gamma_{1}(t, s)=\int \bar{g}_{t}(u)\left(\int_{x \geq u} q(x) d G_{s}(u)\right) d G_{t, s}(u, v), \gamma(t, s)=\gamma_{1}(t, s)+\gamma_{1}(s, t)
$$




\section{Covariance function of two processus}

In some applications, we may be led to simultaneously consider two or several processes of the kind (1). In this case, their covariance function may be useful. Consider

$$
\beta_{n, 2}(t)=\frac{1}{\sqrt{n}} \sum_{j}\left\{G_{t, n}\left(Y_{j}(t)\right)-G_{t}\left(Y_{j}(t)\right)\right\} q_{1, t}\left(Y_{j}(t)\right)
$$

and

$$
\beta_{n, 1}(t)=\frac{1}{\sqrt{n}} \sum_{j}\left\{G_{t, n}\left(Y_{j}(t)\right)-G_{t}\left(Y_{j}(t)\right)\right\} q_{2, t}\left(Y_{j}(t)\right)
$$

We will have the result

Proposition 2. If the two processes are both asymptotically tight and converge in finite-distribution, then their limiting Gaussian processes have the following covariance

$$
\Gamma_{3}(t, s)=g\left(q_{1, t}, q_{2, s}, t, s\right)-\left(\left(E_{t} G_{t} q_{1}\right)\left(E_{s} G_{s} q_{2}\right)+\left(E_{t} G_{t} q_{1}\right)\left(E_{s} G_{s} q_{2}\right)\right),
$$

and

$$
g\left(q_{1, t}, q_{2, s}, t, s\right)=g_{1}\left(q_{1, t}, q_{2, s}, t, s\right)+g_{1}\left(q_{1, s}, q_{2, t}, s, t\right)
$$

with

$$
g_{1}\left(q_{1, t}, q_{2, s}, t, s\right)=\int\left(\int_{x \geq u} q_{1, t}(u) d G_{t}(u)\right)\left(\int_{x \geq v} q_{2, s}(v) d G_{s}(v)\right) d G_{t, s}(u, v)
$$

\section{A useful tool}

We give here a useful lemma on which, is be based the asymptotic finite-distribution normality of the processes involved here. It will be enough to describe it in the two dimensional case. A generalization to the k-dimensional case is straightforward. We have

Lemma 1. Let $\left(X_{i}, Y_{i}\right), i=1,2, \ldots$, be independent observations of a random vector $(X, Y)$ with joint distribution function $G(x, y)=P(X \leq x, Y \leq y)$, and margins $G_{1}(x)=G(x,+\infty)$ and $G_{2}(y)=G(+\infty, y)$. Let, for each $n \geq 1, \varepsilon_{1, n}$ and $\varepsilon_{2, n}$ be the quantile processes based respectively on $G_{1}\left(X_{1}\right), G_{1}\left(X_{2}\right), \ldots, G_{1}\left(X_{n}\right)$, and on $G_{2}\left(Y_{1}\right), G_{2}\left(Y_{2}\right), \ldots, G_{2}\left(Y_{n}\right)$. Then $\varepsilon_{n}=\left(\varepsilon_{1, n}, \varepsilon_{2, n}\right)$ converges in distribution to a Gaussian process $\varepsilon=\left(\varepsilon_{1}, \varepsilon_{2}\right)$ in $\left(\ell^{\infty}([0,1])\right)^{2}$ such that each $\varepsilon_{i}$ is a standard Brownian bridge.

Proof. Let for each $n \geq 1, \alpha_{1, n}$ and $\alpha_{2, n}$ be the empirical processes based respectively on $G_{1}\left(X_{1}\right), G_{1}\left(X_{2}\right), \ldots, G_{1}\left(X_{n}\right)$ and on $G_{2}\left(Y_{1}\right), G_{2}\left(Y_{2}\right), \ldots, G_{2}\left(Y_{n}\right)$. We have (see [9], p.584) that $\alpha_{i, n}(s)=-\varepsilon_{i, n}(s)+o_{P}(1)$ uniformly in $s \in(0,1)$, which gives

$$
\varepsilon_{n}(s, t)=\left(\varepsilon_{1, n}(s), \varepsilon_{2, n}(t)\right)=-\left(\alpha_{1, n}(s), \alpha_{2, n}(t)\right)=o_{P}(1),
$$

uniformly in $(s, t) \in(0,1)^{2}$. Now let us consider the functional empirical process $\alpha_{n}$ based on the $Z_{i}=\left(G_{1}\left(X_{i}\right), G_{2}\left(Y_{i}\right)\right)$, that is

$$
\alpha_{n}(f)=\frac{1}{\sqrt{n}} \sum_{j=1}^{n} f\left(Z_{i}\right)-\mathbb{E} f\left(Z_{i}\right)
$$

for a real function defined on $(0,1)^{2}$ such that $\operatorname{Ef}\left(Z_{i}\right)^{2}<\infty$. We have by the classical results of empirical process that $\left\{\alpha_{n}(f), f \in F\right\}$ converges to a Gaussian process $\{G(f), f \in F\}$ whenever $F$ is a donsker class. It follows that $\left\{\alpha_{n}\left(1_{C}\right)\right.$, $C \in C\}$ converges to a Gaussian process $\left\{G\left(1_{C}\right), C \in C\right\}$ whenever $C$ is a Vapnik-Cervonenkis class (VP-class). But $C=\left\{1_{[0, s] \times[0, t]},(t, s) \in(0,1)^{2}\right\}$ is a $V P$-class of index not greater of 2 . (see [10] for $V P$-classes use to empirical processes). Thus, putting $f_{s, t}=1_{[0, s] \times[0, t]}$, we have

$$
\alpha_{n}(s, t) \equiv \alpha_{n}\left(f_{s, t}\right) \rightsquigarrow \mathbb{G}\left(f_{s, t}\right) \equiv \mathbb{G}(s, t)
$$

in $\left(\ell^{\infty}([0,1])\right)^{2}$, where $\rightsquigarrow$ stands for the weak convergence. Now, by using the Skorohod-Wichura-Dudley Theorem, we are entitled to suppose that we are on a probability space such that

$$
\sup _{(s, t) \in(0,1)^{2}}\left|\alpha_{n}\left(f_{s, t}\right)-\mathbb{G}\left(f_{s, t}\right)\right| \rightarrow_{P} 0 .
$$


Now, put $f_{1, s}=1_{[0, s] \times[0,1]}, f_{2, t}=1_{[0,1] \times[0, t]}, G_{1}(s)=G_{1}\left(f_{1, s}\right)$ and $G_{2}(t)=G_{1}\left(f_{2, t}\right)$. We have

$$
\alpha_{n}\left(f_{i, s}\right)=\alpha_{1, n}(s)=\mathbb{G}_{1}(s)+o_{P}(1),
$$

uniformly in $s \in(0,1)$. We finally have

$$
\alpha_{n}(s, t)=\left(\mathbb{G}_{1}(s), \mathbb{G}_{2}(t)\right)+o_{P}(1),
$$

uniformly in $(s, t) \in(0,1)^{2}$. Clearly, $\left(G_{1}(s), G_{2}(t)\right)$ is a Gaussian process and each $G_{i}$ is the standard Brownian bridge.

Application 1. Let us consider the two-dimensional distribution $\beta_{n}\left(t_{1}, t_{2}, a\right)$ like in $(6)$, which is

$$
\frac{1}{\sqrt{n}}\left\{a_{1} \sum_{j=1}^{n}\left\{G_{t_{1}, n}\left(Y_{j}\left(t_{1}\right)\right)-G\left(Y_{t_{2}}\right)\right\} q_{t_{1}}\left(Y_{j}\left(t_{1}\right)\right)+a_{1} \sum_{j=1}^{n}\left\{G_{t_{2}, n}\left(Y_{j}\left(t_{2}\right)\right)-G\left(Y_{t_{2}}\right)\right\} q_{t_{2}}\left(Y_{j}\left(t_{2}\right)\right)\right\} .
$$

Using the notations around (6), we have

$$
\begin{aligned}
& \beta_{n}\left(t_{1}, t_{2}, a\right)=a_{1} N_{1}\left(q_{t_{1}}, t_{1}\right)+a_{2} N_{1}\left(q_{t_{2}}, t_{2}\right)+o_{P}(1) \\
= & \int_{0}^{1}\left\{a_{1} G_{1}(s) q_{t_{1}}(s)+a_{2} G_{2}(s) q_{t_{2}}(s)\right\} d s+o_{P}(1), \\
\rightarrow & N\left(a_{1}, a_{2}\right)=\int_{0}^{1}\left\{a_{1} G_{1}(s) q_{t_{1}}(s)+a_{2} G_{2}(s) q_{t_{2}}(s)\right\} d s,
\end{aligned}
$$

which is a Gaussian random variable.

\section{Conclusion}

We have entirely described the weak law of empirical stochastic processes like (1) as well that of such processes and a functional emprical processes. Such results have potential powerful applications in deriving uniform time-dependent L-statistics as done in Lo and Sall [7], where the time-dependant general poverty index is studied. Applications of our results in Actuarial Sciences are under way.

\section{References}

[1] Helmers, R. and Ruymgaart, F.H., 1988. Asymptotic normality of generalized L-statistics with unbounded Scores. J. statist. Plann. Inference 19, 43-53.

[2] Helmers, R., Janssen, P. and Serfling, R., 1988. Glivenko- Cantelli Properties of some generalized empirical df's and strong convergence of generalized L-Statistics. Probab. Theory Related Fields., 79, 75-93.

[3] Helmers, R., Janssen, P. and Serfling, R., 1990. Berry-Esséen and bootstrap results for generalized L-statistics. Scand. J. Statist., 17, 65-77.

[4] Puri, M.L., Greselin, F. and Zitikis, R., 2009. L-functions, processes, and statistics in measuring economic inequality and actuarial risks. Statistics and Its Interface, $\mathbf{0}(1)$.

[5] Lo, G.S., 2009. Asymptotic Representation Theorems for poverty indices. Submitted.

[6] Lo, G.S. and Sall, S.T., 2009. On some empirical process. Available at : http://www.ufrsat.org/perso/gslo/empstopro.pdf

[7] Lo, G.S. and Sall, S.T., 2009. The Asymptotic Laws of the Time-dependent General Poverty Index and Applications. Available at : http://www.ufrsat.org/perso/gslo/sall-lo00210.pdf

[8] Lo, G.S., Sall. S. and Seck, C.T., 2009. Une théorie asymptotique des indicateurs de pauvreté. C.R. Math. Rep. Acad. Sci. Canada,2(31), 45-52.

[9] Shorack, G.R. and Wellner J.A., 1986. Empirical Processes with Applications to Statistics, wiley-Interscience, NewYork.

[10] van der Vaart A.W. and Wellner, J.A., 1996. Weak Convergence and Empirical Processes With Applications to Statistics. Springer, New-York.

[11] Sall, S.T. and Lo, G.S., 2009. Uniform Weak Convergence of the Time-dependent Poverty Measure for Continuous Longitudinal Data. Braz. J. Probab. Statist.. 24, 457-467. 